\title{
Retour sur le drame de l'île des Diamants : l'investissement immobilier des grands projets urbains à Phnom-Penh
}

\section{Gabriel Fauveaud}

\section{Q OpenEdition}

1 Journals

\section{Édition électronique}

URL : http://journals.openedition.org/echogeo/12367

DOI : 10.4000/echogeo. 12367

ISSN : 1963-1197

Éditeur

Pôle de recherche pour l'organisation et la diffusion de l'information géographique (CNRS UMR 8586)

Référence électronique

Gabriel Fauveaud, «Retour sur le drame de l'île des Diamants : l'investissement immobilier des grands projets urbains à Phnom-Penh », EchoGéo [En ligne], Sur le Vif, mis en ligne le 03 mai 2011, consulté le 03 mai 2019. URL : http://journals.openedition.org/echogeo/12367 ; DOI : 10.4000/echogeo.12367

Ce document a été généré automatiquement le 3 mai 2019.

\section{(c) (i) (9)}

EchoGéo est mis à disposition selon les termes de la licence Creative Commons Attribution - Pas d'Utilisation Commerciale - Pas de Modification 4.0 International 


\title{
Retour sur le drame de l'île des Diamants : l'investissement immobilier des grands projets urbains à Phnom-Penh
}

\author{
Gabriel Fauveaud
}

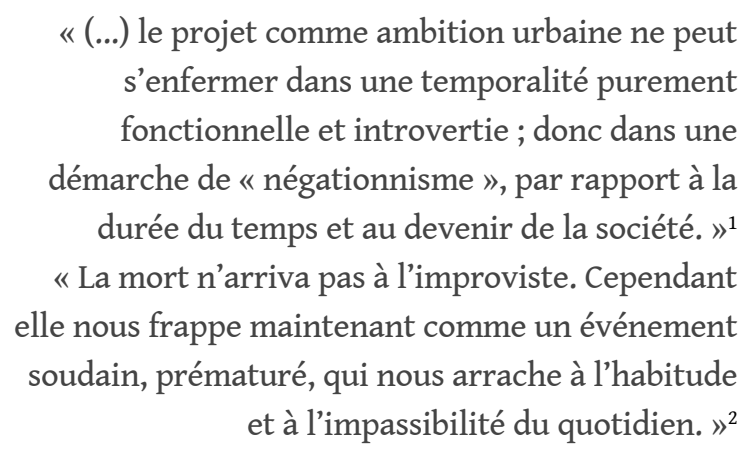

Le 22 novembre 2010 un mouvement de foule causa la mort de plus de 350 personnes sur le pont suspendu de l'île des Diamants, grand projet urbain privé actuellement en construction à Phnom-Penh (illustration 1). Le drame eut lieu pendant la Fête des Eaux qui accueillait cette année près de 3 millions de personnes pendant trois jours au sein de la capitale cambodgienne qui compte 1300000 habitants $^{3}$ environ. La Fête des Eaux célèbre le retournement des eaux du fleuve Tonlé Sap engendré par les crues et décrues du fleuve Mékong. Le Tonlé Sap alimente de novembre à mai le Mékong, et de mai à novembre le lac Tonlé Sap, véritable cœur agricole du Cambodge.L'attraction phare des célébrations est la course de pirogues qui se déroule sur le site des Quatre Bras ${ }^{4}$ et où concourent des équipes venant de l'ensemble du royaume. Le roi du Cambodge préside la cérémonie du haut de l'appontement royal pendant les trois jours que dure la compétition $^{5}$. De nombreux événements ont lieu au sein des espaces publics de la ville. L'île des Diamants accueillait cette année des concerts de musique et diverses attractions. Un grand nombre de spectateurs se rendirent sur l'île au cours des festivités. La compagnie privée gestionnaire de l'île des Diamants était en charge de la sécurité sur le 
site. L'accumulation de spectateurs sur un des deux ponts d'accès provoqua un engorgement qui empêcha alors tous déplacements pendant plusieurs heures. Un accès de panique déclencha le mouvement de foule meurtrier. Cet événement dramatique questionne le rôle des acteurs publics et privés dans la transformation des espaces urbains contemporains de Phnom-Penh, et la capacité des promoteurs immobiliers à produire une ville pérenne.

2 En partant de la tragédie de l'île des Diamants, cet article se propose de revenir sur l'évolution des modes de construction de la capitale cambodgienne. Notre première partie évoquera l'internationalisation des modes de construction en Asie du Sud-Est et au Cambodge. Nous montrerons que l'aménagement de Phnom-Penh à partir du début des années 1990 se caractérise par un retrait de la planification urbaine et une multiplication de «projets urbains » qui conditionnent notamment le rapport entre les investisseurs privés et les acteurs institutionnels. Dans notre seconde partie, l'exemple de l'île des Diamants nous permettra d'évoquer certaines grandes tendances de l'urbanisation à Phnom-Penh. Nous montrerons comment l'évolution du discours sur la ville moderne génère des antagonismes au sein de la politique urbaine actuelle. Notre thèse ici sera que l'investisseur privé des grands projets immobiliers ne vend pas des espaces urbains mais bien un produit d'investissement.

\section{Illustration 1 - Construction des grands projets et évolution des espaces urbains}

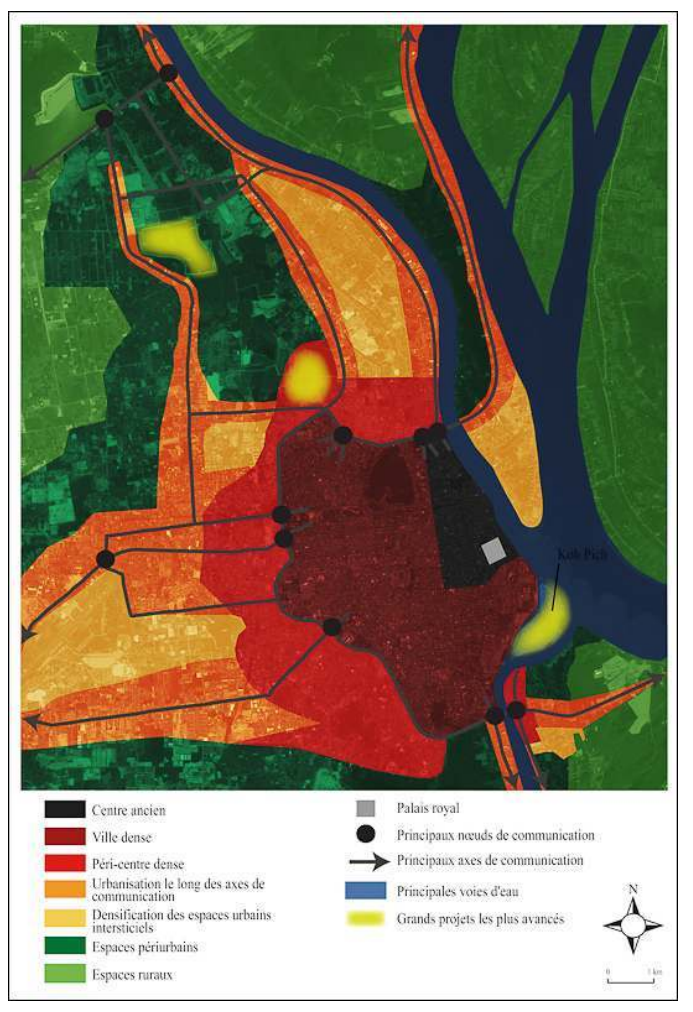

Source : image SPOT 2007, Bureau des Affaires Urbaines de Phnom-Penh ; réalisation : G. Fauveaud. 


\section{Internationalisation des modes de construction et généralisation des « projets urbains »}

\section{L'internationalisation des modes de construction en Asie du Sud-Est}

Depuis les années 1980, de nombreuses recherches s'intéressent aux rôles des villes mondiales dans la réorganisation géographique des flux de capitaux dont les espaces urbains sont le centre de synergie (Harvey, 1985 ; Friedman, 1986 ; Smith, 1990 ; Sassen, 1992). L'ouverture des espaces urbains sud-est asiatiques à l'économie mondiale n'est pas un phénomène récent (Ginsburg, 1955 ; Bishop, Phillips et Yeo, 2003). A partir des années 1970, l'émergence des « Dragons Asiatiques » dont Singapour est l'exemple emblématique (Goldblum, 1988; Guillot, 2004) marque un tournant historique dans les processus d'intégration de l'Asie du Sud-Est à l'économie mondiale. Au cours des années 1980, Bangkok et Jakarta sont à leurs tours concernées par l'internationalisation des flux financiers et des modes de construction. La croissance de ces centres urbains amène de nouveaux questionnements sur les recompositions métropolitaines au sein de la région dont la « Desakota » est sûrement la modélisation spatiale la plus aboutie (Mc Gee, 1991). Dans la continuité de ces travaux, Dick et Rimmer (1998) portent leur attention sur la place des acteurs régionaux et mondiaux dans la construction des villes asiatiques. Pour eux, les travaux des urbanistes, des concepteurs, des spécialistes de l'immobilier et des architectes entrainent une convergence des modèles de construction des villes sud-est asiatiques avec ceux des villes des pays développés ${ }^{6}$. L'émergence de compagnies à dimension internationale accélère le transfert des modèles urbains à l'échelle régionale (Scott, 2001; Haila, 2002; Guillot, 2005). Ces nouvelles dynamiques relancent les interrogations sur les liens entre l'urbanisme et les évolutions socio-économiques de ces espaces urbains (King, 2004). Constater que la mondialisation participe à la transformation des espaces urbains en Asie du Sud-Est - propos qui tient presque du lieu commun aujourd'hui - masque néanmoins des réalités locales plus complexes.

\section{Urbanisme et projets politiques à Phnom-Penh}

La mise en place de modèles urbains à Phnom-Penh est visible à partir de son avènement en tant que nouvelle capitale cambodgienne entre 1863 et $1866 .{ }^{7}$ L'espace urbain devient l'illustration des stratégies politiques et idéologiques qui participent à la construction d'une identité nationale à partir de la capitale.

5 La modernisation des pays colonisés, qui justifie notamment la présence des Français au Cambodge et plus largement en Indochine, doit passer par la mise en valeur préalable d'une certaine «tradition locale». Comme le rappelle Wright, l'urbanisme indochinois soutient l'entreprise colonisatrice en construisant des espaces et bâtiments urbains qui opèrent une synthèse entre une culture locale traditionnelle et une modernité métropolitaine (Wright, 1987). Les concepts de «tradition » et de «modernité » en tant que construits idéologiques s'imposent au cours des années 1910 dans les projets urbains Indochinois. Edwards (2007) souligne le rôle d'urbanistes français au Cambodge tel que Hébrard ${ }^{8}$ - dont les principales réalisations se trouvent à Hanoi et Hô Chi Minh - dans la construction d'une nouvelle identité nationale à partir de Phnom-Penh. 
6 A partir de l'indépendance en 1953, et surtout après l'élection du Sangkum Reastr Niyum 9 en 1955, Phnom-Penh fait l'objet de grands travaux qui doivent illustrer la nouvelle identité nationale (Molyvann, 2003). Cette transformation de l'espace urbain s'accompagne d'une réflexion architecturale - baptisée rétrospectivement New Khmer - qui affirme le renouveau de la culture cambodgienne en rupture avec son passé colonial. Si le contexte politique n'est plus le même, ce processus s'inscrit dans la continuité de la période précédente. En effet, le New Khmer évoque à la fois l'architecture angkorienne classique et l'architecture du Mouvement Moderne (Grant Ross et Collins, 2006). La redéfinition de la culture cambodgienne à partir de l'indépendance se réalise dans la synthèse d'une «tradition nationale» et d'une «modernité internationale». Le couple «tradition/ modernité " participe à la même volonté de reformuler une identité nationale dont la capitale cambodgienne est une fois encore le support premier. La modernité n'évoque cependant plus le rapport avec la métropole, mais plutôt l'appartenance à une communauté politique et culturelle mondiale illustrée par l'engagement de Sihanouk dès 1955 dans le groupement des pays non-alignés.

7 La prise de pouvoir des Khmers Rouges le 17 avril 1975 marque une rupture historique dans les processus de développement de la capitale cambodgienne ${ }^{10}$. A la fin de l'année 1978, l'armée vietnamienne met en déroute l'armée Khmère Rouge et occupe le pays jusqu'en 1989. La signature des Accords de Paris le 23 octobre 1991 a pour but, selon les termes onusiens, « le règlement global du conflit cambodgien ». Ils préfigurent la tenue d'élections libres et représentent le point de départ d'une intervention massive de la communauté internationale afin de « reconstruire » le pays, notamment en l'intégrant au sein de l'économie régionale et internationale. L'ouverture du pays à l'aide internationale et aux investissements étrangers transforme les dynamiques de construction des espaces urbains de Phnom-Penh.

\section{Fragmentation institutionnelle et multiplication des projets immobiliers}

8 A partir du début des années 1990, la multiplication de grands projets de constructions privés et l'édification d'infrastructures urbaines financées par des organisations de coopérations internationales annoncent une transformation des logiques de fabrication des espaces urbains.

9 Phnom-Penh est parfois directement (réhabilitation, consolidation et développement des infrastructures; politique de l'habitat; gestion du patrimoine; renforcement institutionnel) ou indirectement concernée par des projets de développement mis en place par des institutions internationales et des organisations non gouvernementales. Au sein de ces projets, la coopération institutionnelle se fait principalement de manière bilatérale entre un acteur du développement et une ou plusieurs institutions locales (ministères, agences gouvernementales, services de la municipalité). Trouver un interlocuteur politique local devient un préalable indispensable à la mise en place des projets de développement. L'entrée dans l'environnement politique d'acteurs allogènes qui apportent avec eux des outils techniques de l'expertise internationale accroît la fragmentation institutionnelle locale (Mosse, 2007). L'appropriation des ressources (humaines, techniques et financières) par les institutions cambodgiennes génère des enjeux de pouvoir importants au sein des ministères et de la municipalité qui ne favorisent pas une synergie décisionnelle. 
10 Parallèlement, la stabilité politique et économique entraîne à partir du milieu des années 1990 une croissance exponentielle des investissements privés, et particulièrement des projets immobiliers. Les investisseurs des grands projets de construction sont confrontés à un État extrêmement centralisé d'un point de vue politique, mais particulièrement fragmenté en termes de compétences et de moyens techniques. Comme pour les organisations internationales, l'interlocuteur politique local devient un élément clé ${ }^{11}$ de la réalisation des projets urbains, facteur qui prend le pas sur les modalités techniques de planification et de construction. En d'autres termes, les investisseurs dépensent plus d'énergie, de temps et d'argent à assurer politiquement leurs investissements ${ }^{12}$ qu'à intégrer leurs projets au sein d'une ville planifiée. Loin de pâtir de cette situation, les investisseurs privés ont su inclure ces réalités dans leurs projets d'investissement, souvent de manière informelle.

11 La construction de grands projets urbains à Phnom-Penh depuis le début des années 2000 souligne ainsi l'évolution de la politique édilitaire locale qui donne la priorité aux projets urbains plutôt qu'à la planification urbaine. Cette rupture historique est la conséquence de la destruction des structures institutionnelles pendant le régime Khmer Rouge mais correspond aussi à une domination croissante des logiques de marché dans la construction actuelle des espaces urbains.

\section{Nouveaux modes de construction des espaces urbains à Phnom-Penh}

\section{Un projet global pour une nouvelle centralité}

12 L'île des Diamants apparaît à partir de la fin des années 1970. Le site s'est formé par dépôts alluvionnaires principalement, puis par un travail de remblayage par pompage du sable du Tonlé Bassac engagé de 2006 à 2008 par l'investisseur privé. Le projet est développé par l'Overseas Cambodian Investment Corporation (OCIC), filiale de la Canadia Bank, principal financeur du projet. L'OCIC obtint un bail emphytéotique de 99 ans en 2006 auprès du gouvernement pour l'exploitation de l'île, puis devint propriétaire définitive en 2009. Le schéma directeur du projet fut approuvé en 2006 par le Ministère de l'Aménagement du Territoire, de l'Urbanisme et de la Construction et la Municipalité de Phnom-Penh. Avant le ralentissement économique de 2008, l'achèvement total du projet était prévu en 2017. Les 100 hectares de terrain aménagés comprennent des espaces résidentiels et commerciaux, des bureaux, des halls de conférences et des espaces récréatifs. 


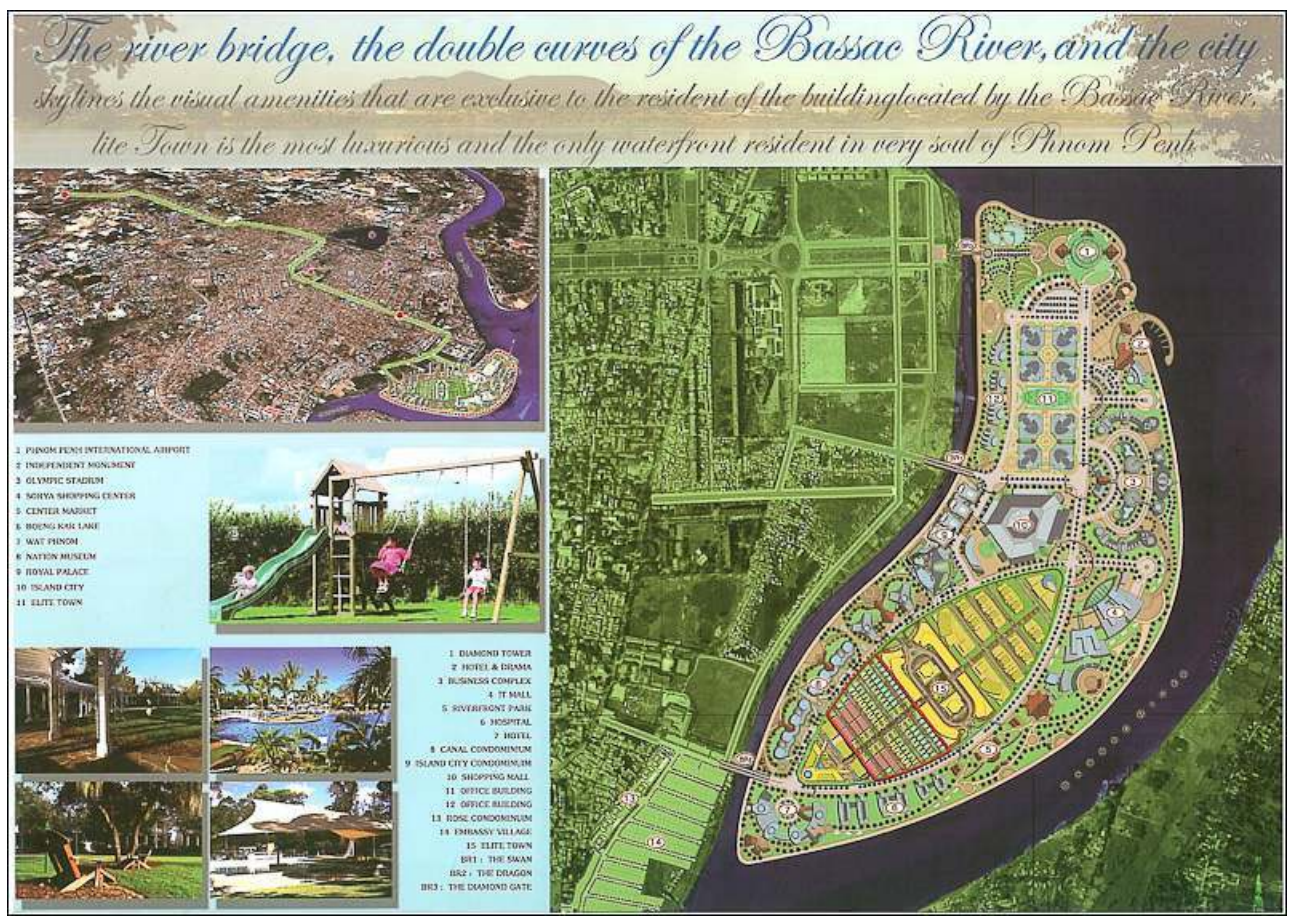

Source : OCIC.

13 Une fois le projet réalisé, l'OCiC prévoit une affluence de 100000 personnes par jour, chiffre qui comprend habitants, travailleurs et visiteurs. De 30000 à 40000 personnes logeront de manière permanente dans la partie résidentielle fermée appelée «Elite Town » qui offrira une large gamme de services aux habitants. Le projet offre par ailleurs des espaces accessibles au public, en vue dans un premier temps d'y «attirer les gens pour promouvoir le projet $»^{13}$. Dès l'ouverture des espaces récréatifs, une partie de la population investit le lieu qui devint assez rapidement populaire, comme en témoignent l'occupation des berges en fin de journée et l'organisation de cérémonies privés. Plus largement, la situation de l'île des Diamants dans la partie sud de la ville-centre illustre un redéploiement de la centralité urbaine de l'ancien quartier colonial vers le sud du palais royal (illustrations 1). La construction d'un hôtel Sofitel, d'une école internationale, de trois tours de logements par l'entreprise coréenne Posco E\&C et d'espaces résidentiels privés atteste de la transformation rapide de cette partie de la ville (illustration 3 ). 
Illustration 3 - Représentation publicitaire de la transformation des espaces de berges du Tonlé Bassac qui font face à l'île des Diamants

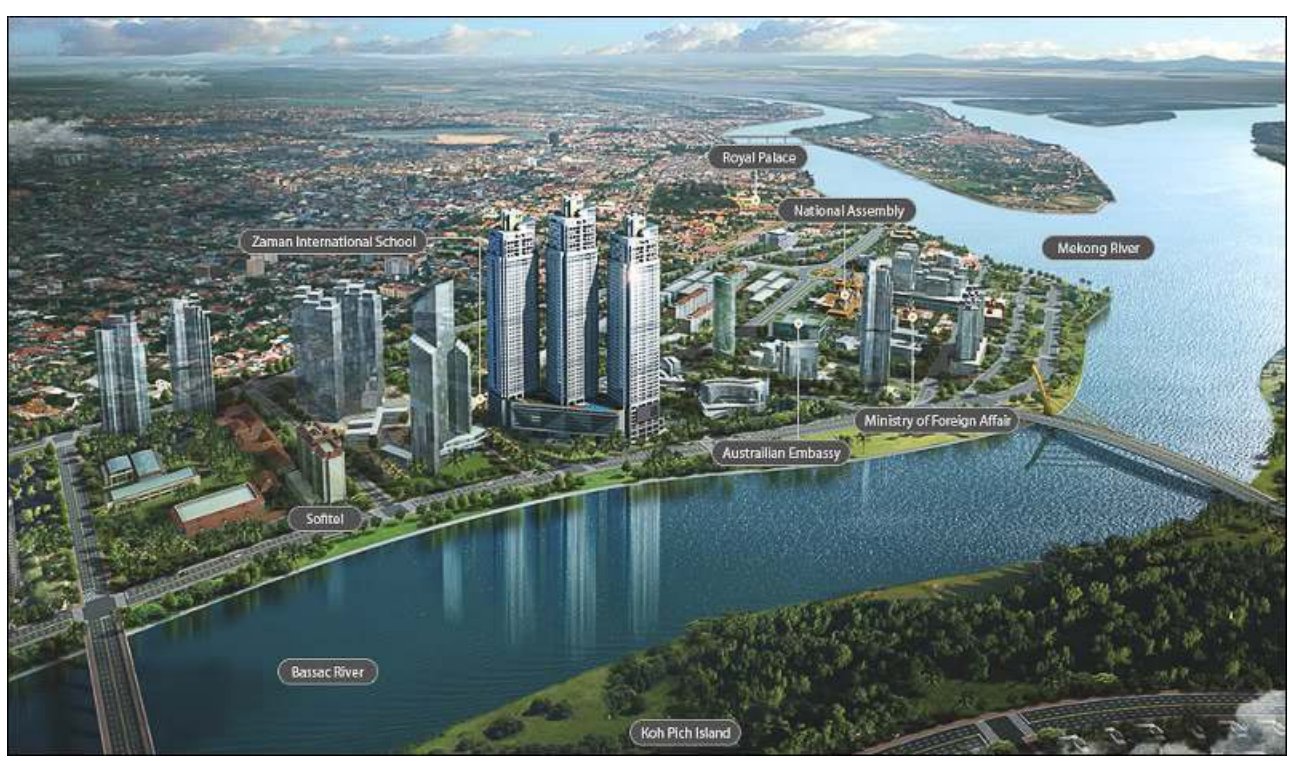

Source : Posco E\&C.

14 L'île des Diamants représente une des grandes tendances de l'urbanisation de Phnom-Penh : celle des grands projets urbains privés qui mettent en avant une urbanité allochtone, signe d'une nouvelle modernité urbaine à destination d'une population étrangère et locale solvable. Cette évolution de la production des espaces urbains à Phnom-Penh s'appuie notamment sur une reformulation du discours sur la ville moderne que nous évoquions précédemment.

\section{Marketing urbain et évolution du discours sur la modernité}

Le marketing urbain mis en place par les investisseurs des grands projets urbains s'étançonne sur une réactualisation du couple tradition/modernité. L'utilisation d'une sémiotique évoquant l'internationalisation des modes de construction est systématique. L'emploi de termes comme « international city ", « sky line » ou « world city " (illustration 2) dans les campagnes publicitaires et les représentations graphiques des constructions et des espaces urbains en sont les meilleurs exemples (illustration 3). La multiplication de blogs et de sites Internet dédiés aux grands projets permet une diffusion rapide de ce discours publicitaire. D'un autre coté, les références à l'espace local (illustration 2) sont nombreuses, comme en témoigne un des slogans publicitaires de l'île des Diamants qui évoque le site naturel de Phnom-Penh : "the water culture and beyond». Le couple local/ global vient ainsi remplacer le couple tradition/modernité.

Cette dualisation idéologique de la construction des espaces urbains est assimilée par les acteurs publics et individuels. Pour les autorités locales, la construction de grands projets permet à Phnom-Penh d'accéder à une modernité globale et de participer à la concurrence interurbaine régionale et mondiale (Harvey, 1985). Pour les citadins, acquérir un bien immobilier au sein d'un grand projet est gage d'une bonne situation sociale et économique ${ }^{14}$. Pour les entrepreneurs des grands projets, l'assimilation du marketing urbain par les autorités locales et les potentiels acheteurs cambodgiens 
conditionne la réussite de leur projet ${ }^{15}$. La modernisation de Phnom-Penh, qui lui permettrait de s'élever au rang des autres capitales régionales, devient un «projet de ville » dans son ensemble. La spectacularisation de l'île des Diamants durant la Fête des Eaux a permit à l'OCIC de faire une grande publicité de son projet, et aux autorités locales de présenter la modernisation de la ville comme une réalisation politique importante. Comme le note Rosemberg-Lasorgne, « (...) faire accepter le projet de ville c'est aussi et en même temps construire une représentation collective du projet, telle que les habitants prennent en charge le projet, y participent. » (Rosemberg-Lasorgne $1997:$ p. 4). A la fois illustration et matrice du projet de ville, l'île des Diamants se situe au croisement des intérêts des acteurs publics et privés, qui construisent une nouvelle représentation collective de la ville afin de dégager un profit politique pour les uns, un profit financier pour les autres.

Cette « modernité urbaine glocale » évoquée par Swyngedouw et Kaika est caractéristique de la néo-libéralisation des espaces urbains à l'échelle mondiale, réalisée par une « intégration externe » à la globalisation et favorisant une « fragmentation interne » des sociétés locales (Swyngedouw et Kaika, 2005).

\section{Fabriquer la ville par l'investissement immobilier}

$18 \mathrm{Au}$ sein d'une économique caractérisée par une forte spéculation foncière, la rentabilisation de l'investissement immobilier prend le pas sur la volonté d'édifier des espaces urbains pérennes. La construction des grands projets correspond à des stratégies économiques qui interrogent la capacité de ces nouveaux espaces urbains à s'intégrer à la ville existante. Pour reprendre la formule de Choay (2006), les espaces urbains des grands projets sont-ils synonymes d'urbanité?

19 Les grands investisseurs privés s'appuient notamment sur l'essor d'une nouvelle génération de citadins particulièrement perméables aux cultures urbaines diffusées depuis Bangkok, Singapour, Hong-Kong ou Kuala Lumpur par exemple. Ces acheteurs immobiliers locaux participent selon eux, en prenant part au projet, à une sphère vertueuse d'investissement qui engendrera une plus-value à leur propre achat immobilier. Le grand projet urbain suscite ainsi un autre niveau d'investissement local. Les grands investisseurs ne construisent pas la ville vantée sur les publicités, car l'investissement local au sein de ces projets se fait avant tout à des fins spéculatives. Ce n'est pas tant le discours sur la ville moderne qui est intégré par les acteurs locaux mais plutôt le facteur «moderne" du produit immobilier vu comme un gage de bon investissement. "Notre retour sur investissement sera d'autant plus grand si nous adhérons au projet de modernisation de l'espace urbain, qui fera augmenter la demande locale, et donc le prix des logements dans le projet ${ }^{16}$ ». Les acheteurs immobiliers locaux, en adhérant au discours des grands investisseurs, affermissent le grand projet en tant que « lieu symbolique » (Debardieux, 1995). L'île des Diamants s'assimile plus à un produit d'investissement où la dimension symbolique domine, qui à elle seule ne peut permettre à un espace urbain de fonctionner, de s'articuler avec la ville existante, de s'accorder avec les réalités socio-spatiales locales.

20 L'homogénéisation des espaces urbains ne signifie pas l'uniformité des pratiques sociospatiales. Le drame de lîle des Diamants souligne que la pratique de nouveaux espaces urbains peut être dangereuse à partir du moment où les réalités sociales locales ne sont pas prises en compte. Ces antagonismes génèrent des tensions et des incompréhensions 
dans la formation de cette nouvelle urbanité qui émerge au sein d'une société encore en reconstruction, facteur qui n'entre pas dans les stratégies économiques des promoteurs immobiliers.

\section{Conclusion}

21 L'évolution historique des modes de construction illustre une transformation de l'instrumentalisation idéologique et politique des espaces urbains à Phnom-Penh. De 1863 à 1970, la ville sert de support à l'élaboration d'une communauté nationale, soit pour mettre en valeur l'entreprise colonisatrice, soit pour soutenir la reconfiguration politique après l'indépendance. La réouverture du pays à partir du début des années 1990 se caractérise par un retrait de l'État dans la planification urbaine et une domination des logiques de marché dans la production des espaces urbains. Les acteurs privés deviennent ainsi les principaux protagonistes des transformations de la ville. Ils s'appuient sur un marketing urbain qui diffuse à travers une sémiotique des espaces globaux une nouvelle perception de la modernité urbaine, réinterprétée et relayée par les institutions et les investisseurs individuels locaux. Les grands projets, par la dimension des aménagements construits et l'importance des flux de capitaux engendrés, sont des vecteurs importants, voir stratégiques, de ces processus.

La place du projet urbain dans la construction contemporaine de Phnom-Penh mérite d'être resituée au sein d'une analyse plus globale. Certaines tendances que nous venons de décrire sont caractéristiques de l'évolution des villes néo-libérales (Brenner N. et Theodore N., 2002; Peck et Tickell, 2002). L'apparition de nouveaux acteurs urbains, la concurrence inter-urbaine, l'importance de l'échelle régionale et la recherche incessante de nouveaux marchés par les investisseurs privés touchent des espaces auparavant exclus de la mondialisation (Franck M. et Goldblum C., 2007). Conséquences de ces dynamiques, la prévalence du politique sur la gestion technique, la fragmentation institutionnelle ainsi que la primauté des motivations d'ordre économique révèlent une construction de la ville du global vers le local et de l'élite économico-institutionnelle vers les citadins. Ces nouveaux modes de construction des espaces urbains à Phnom-Penh, qui servent avant tout l'investisseur privé et certains partenaires institutionnels, se détachent des réalités socio-économiques locales.

La reconstruction politique, économique et sociale du Cambodge n'est pas achevée. Les investissements privés se réalisent au sein de cadres fonciers, juridiques et urbanistiques flous qui favorisent la concussion et l'imbrication des acteurs institutionnels et des investisseurs privés, lorsqu'ils ne se confondent pas. Le drame de l'île des Diamants montre le risque de confondre la fabrication de l'espace urbain avec l'investissement immobilier, la ville devenant une agrégation de "projets partiels des marchands d'espaces » plutôt que « l'espace social effectué » (Lefebvre, 2000). 


\section{BIBLIOGRAPHIE}

Bishop R., Phillips J., Yeo W.-W., 2003. Perpetuating Cities: Excepting Globalization and the Southeast Asia Supplement. In Bishop R., Phillips J., Yeo W.-W. ed., 2003. Postcolonial Urbanism. Southeast Asian Cities and Global Processes, Routledge, New-York, p. 1-36.

Brenner N. et Theodore N., 2002. Cities and the Geographies of 'Actually Existing Neoliberalism'; In Brenner N. et Theodore N. eds. Spaces of Neoliberalism: Urban Restructuring in North America and Western Europe, Oxford, Blackwell, p. 2-32.

Choay F., 2006. Le règne de l'urbain et la mort de la ville. In Choay F. Pour une anthropologie de l'espace, Paris, Seuil, p. 165-198;

Debarbieux B., 1995. Le lieu, le territoire et trois figures de rhétorique. L'Espace Géographique, p. $97-112$

Dick H.W. et Rimmer P.J., 1998. Beyond the Third World City: The New Urban Geography of South-east Asia. Urban Studies, vol. 35, n . 12, p. 2303 - 2320.

Edwards P., 2007. Cambodge. The Cultivation of a Nation, 1860 - 1945. Honolulu, Universtity of Hawaii Press, $349 \mathrm{p}$.

Franck M. et Goldblum C., 2007. Les villes aux marges de la métropolisation en Asie du Sud-Est. Espace géographique, tome 36, p. 229-236.

Friedmann J., 1986. The World City Hypothesis. Development and Change, n 17, p. 69-84.

Ginsburg N.S., 1955. The Great City in Southeast Asia. The American Journal of Sociology, vol. 60, n ० 5, p. 455-462

Goldblum C., 1988. Singapour 1819-1986 : émergence de la ville moderne et mythe rural. Archipel, vol. 36, p. 227-270.

Grant Ross, H. et Collins D., 2006. Building Cambodia: "New Khmer Architecture" 1953-1970. Bangkok, The Key Publisher Company, 333 p.

Guillot X., 2007. Singapour : l'urbanisation du Sud dans le prisme de la mondialisation. Autrepart, $\mathrm{n}^{\circ} 41$, p. $165-179$.

Guillot X., 2005. Flux économiques, transferts d'expertises et production immobilière haut de gamme en Asie orientale. Géocarrefour, vol. 80, n³ 3, p. 181-191.

Haila A., 2002. State-present capitalism: property and development companies in Singapore. Entreprises et Histoire, $\mathrm{n}^{\circ} 30$, p. 63-72.

Harvey D., 1985. The Urbanization ofCapital: Studies in the History and Theory of Capitalist Urbanisation. Baltimore, TheJohnsHopkinsUniversityPress, $239 \mathrm{p}$.

King A., 2004. Spaces of Global Cultures. Architecture Urbanism Identity. New York, Routledge, 256 p. Lefebvre H., 2000. Espace et politique : le droit à la ville II. Paris, Anthropos, $4{ }^{\text {ème }}$ édition, 174 p. Mc Gee T., 1991. The Extended Metropolis: Settlement Transition in Asia. Honolulu, Universtity of Hawaii Press, 339 p. 
Mosse D., 2007. Notes on the Ethnography of Expertise and Professionals in International Development. conférence Ethnografeast III, Lisbonne, 20-23 juin 2007.

Molyvan V., 2003. Modern Khmer Cities. Phnom-Penh, Reyum, 235 p.

Peck J. et Tickell A., 2002. Neoliberalizing Space. Brenner N. et Theodore N. eds. Spaces of Neoliberalism: Urban Restructuring in North America and Western Europe, Oxford, Blackwell, p. 380-404.

Rosemberg-Lasorne M., 1997. Marketing urbain et projet de ville : parole et représentations géographiques des acteurs. Cybergeo : European Journal of Geography [En ligne], document 32, mis en ligne le 23 octobre 1997.

Sassen S., 1991. The Global City: New York, London, Tokyo. Princeton, Princeton University Press, $397 \mathrm{p}$.

Scott A.J. (dir.), 2001. Global City Regions: Trends, Theory, Policy. Oxford, Oxford University Press, $467 \mathrm{p}$.

Swyngedouw E. et Kaika M., 2005. La production de modernités urbaines « glocales » : explorant les failles dans le miroir. Géographie, Économie, Société, vol. 7, n² 2, p. 155-176.

Smith N., 1990. Uneven Development: Nature, Capital and the Production of Space. Oxford, Basil Blackwell, 219 p.

Wright G., 1987. Tradition in the Service of Modernity: Architecture and Urbanism in French Colonial Policy, 1900-1930. The Journal of Modern History, vol. 59, n² 2 p.291-316.

\section{NOTES}

1. Chesnaux J., 2001. Mémoire et projet urbain. In Paquot T. (dir.), 2001. Le quotidien urbain. Paris, La découverte, $192 \mathrm{p}$.

2. Habermas J. et al., 2005. Dernier salut. In Rue Descartes, $\mathrm{n}^{\circ}$ 48, p. 70-71.

3. Estimation selon le recensement national effectué en 2008 par le National Institut of Statistics du Cambodge.

4. Les «Quatre Bras» désignent la confluence en amont du Tonlé Sap et du Mékong, et la divergence en aval du Mékong et du Tonlé Bassac.

5. Pour une lecture approfondie sur l'événement de la fête des eaux à Phnom-Penh, nous invitons le lecteur à se reporter aux travaux de Leclère A., 1904. La fête des eaux à Phnom-Penh. In Bulletin de l'École française d'Extrême-Orient, tome 4, p. 120-130 et de Pierdet C., 2005. La symbolique de l'eau dans la culture cambodgienne : fête des eaux et projets urbains à Phnom Penh. In Géographie et culture, $n^{\circ} 56$, p.5-22.

6. "In a new phase of rapid technology transfer and economic growth, south-east Asian cities are again showing clear evidence of converging with Western patterns of urbanisation." (Dick et Rimmer, 1998 : p. 2318).

7. Le traité du protectorat de la France au Cambodge est ratifié en 1863. Phnom-Penh devient officiellement capitale du Cambodge en 1866, après que le roi et sa cour s'y installent définitivement. Pour une lecture approfondie sur l'évolution de la capitale cambodgienne depuis ses origines, nous invitons le lecteur à se reporter aux travaux de Lamant P.-L., 1991. La création d'une capitale par le pouvoir colonial: Phnom Penh. In Péninsule indochinoise. Études urbaines. Paris, L'Harmattan, p. 59-102 et de Goulin C., 1966. Phnom Penh - Étude de géographie urbaine. Doctorat de 3e cycle de géographie, Phnom Penh, 242 p. 
8. C'est à partir de 1910 que fut introduite la science de l'urbanisme en France par Henri Prost notamment, proche collaborateur et ami d'Ernest Hébrard. Prost réalisa de nombreux plans d'aménagement urbain au sein des colonies françaises au Maghreb.

9. Traduit habituellement par la «Communauté Socialiste Populaire », le Sangkum Reastr Niyum fut créé par le roi Norodom Sihanouk qui dirigea le pays de 1955 à 1970. Il est le père du roi actuel, Nordom Sihamoni.

10. Le régime Khmer Rouge se basait notamment sur une idéologie anti-urbaine. Les principales villes du pays furent vidées de leurs habitants peu après la chute de Phnom-Penh. Près d'un tiers de la population du pays ne survivra pas à presque quatre années de pouvoir d'un régime aujourd'hui qualifié de "génocidaire ». La majeure partie de l'élite intellectuelle fut par ailleurs assassinée.

11. Entretien réalisé avec la Korean Trade-Investment Promotion Agency (KOTRA) le 12 juin 2009.

12. Le Cambodge est classé $147^{\mathrm{e}}$ sur 183 pays par la Banque Mondiale en terme de risque d'investissement.

13. Entretien du 26 juin 2009 avec Tout Samnang, chargé de projet pour l'OCIC

14. Nous basons ici notre propos sur un peu plus de 500 entretiens auprès de ménages urbains et périurbains réalisés à Phnom-Penh entre 2008 et 2010.

15. Entretien du 26 juin 2009 avec Tout Samnang, chargé de projet pour l'OCIC.

16. Enquête réalisée entre 2008 et 2010 auprès d'une famille dont le fils cadet est un acheteur, comme six autres de ses amis, d'un appartement à Camko City, un des grands projets actuellement en construction.

\section{RÉSUMÉS}

Cet article se propose de revenir sur la mort, suite à un mouvement de foule qui eut lieu le 22 novembre 2010, de plus de 350 personnes sur le pont d'un grand projet urbain privé actuellement en construction à Phnom-Penh. Il s'agira pour nous, à partir de ce drame marquant, de souligner certaines dynamiques contemporaines de la construction des espaces urbains de la capitale cambodgienne. A travers le grand projet de l'île des Diamants, nous souhaitons interroger le rôle des grands promoteurs immobiliers dans la fabrication de la capitale. Pour ce faire, nous évoquerons l'internationalisation des modes de construction en Asie du Sud-Est et au Cambodge qui se caractérise par un retrait de la planification urbaine et une multiplication des projets urbains privés. L'exemple de l'île des Diamants nous permettra d'illustrer certaines grandes tendances de l'urbanisation à Phnom-Penh. Nous montrerons comment l'évolution du discours sur la ville moderne génère des antagonismes au sein de la politique urbaine actuelle, et interrogerons la capacité des grands investisseurs immobiliers à fabriquer des espaces urbains pérennes.

Through this article, we would like to underline few main dynamics of the contemporary urban development of Phnom-Penh after more than 350 people died on Diamonds Island, a new private large scale project under construction. The Diamonds Island project questions the role of the large scale real-estate investors in the fabrication of the urban space. We will get onto the evolution of the internationalization of the production of the urban space. By this end, we will be able to replace the "urban project" within the institutional and historical local reality. Through 
the particular example of the Diamonds Island, we will approach the evolution of the urban modernity understood as a marketing strategy. Finally, the Diamond Island tragedy reveals the antagonisms of the current urban politic and interrogates the capacity of the private investors to construct a sustainable city.

INDEX

Mots-clés : grand projet urbain, Île des Diamants, investissement immobilier, Phnom-Penh, privatisation de l'espace ; développement urbain

Keywords : Diamonds Island, Phnom-Penh, privatization of space, Real-estate investment, urban development, urban large scale project

\section{AUTEUR}

\section{GABRIEL FAUVEAUD}

Gabriel Fauveaud, gabriel.fauveaud@gmail.com, est doctorant sous la direction du Professeur Thierry Sanjuan, Laboratoire PRODIG (UMR8586), École Doctorale de Géographie de Paris, Université Paris 1 Panthéon-Sorbonne. 